\title{
Research Article \\ Extended Surface of Materials as a Result of Chemical Equilibrium
}

\author{
Walerian Arabczyk, Rafał Pelka, and Izabella Jasińska \\ Institute of Chemical and Environment Engineering, West Pomeranian University of Technology in Szczecin, \\ Pułaskiego 10, 70322 Szczecin, Poland \\ Correspondence should be addressed to Izabella Jasińska; jasinska@zut.edu.pl
}

Received 24 June 2014; Revised 2 October 2014; Accepted 11 October 2014; Published 4 November 2014

Academic Editor: Zhenbo Wang

Copyright (C) 2014 Walerian Arabczyk et al. This is an open access article distributed under the Creative Commons Attribution License, which permits unrestricted use, distribution, and reproduction in any medium, provided the original work is properly cited.

\begin{abstract}
A system consisting of at least two components was considered. In this system, nanocrystalline material is formed at high temperature, at which diffusion does not limit the mass transport. The structure results from establishing an equilibrium between surface and volume of the crystallites and their surroundings in isothermal-adiabatic conditions. The surface of each crystallite is covered with another substance. On the basis of the performed energy-balance calculations it was concluded that the reduction in the surface area is associated with a decrease in the surface coverage degree and thus with the necessity to provide energy to the system in order to remove chemisorbed atoms. An increase in the temperature of a nanocrystalline substance to a temperature higher than the preparation temperature results in the formation of a new state of equilibrium. At temperatures below the maximum temperature only the equilibrium between the gas phase and the surface exists.
\end{abstract}

\section{Introduction}

There is a common view that substances with a strongly extended surface are not in a state of chemical equilibrium. The surface energy, $\gamma$, of a solid is associated with the presence of unsaturated bonds to atoms located on a solid surface. Substances in nature tend to approach an energy minimum, and this is achieved by decreasing the surface to volume ratio, $S / V[1-4]$.

Catalysts are examples of materials with a strongly extended surface. The influence of temperature and a gaseous atmosphere on the specific surface area and the metal dispersion on catalyst support has been described in numerous papers [5-7]. The changes in surface area and metal dispersion during annealing at a given temperature have been studied. Based on the data from these studies, it can be concluded that neither the surface area nor the metal dispersion reaches a zero value, despite prolonged exposure to a high temperature. One example is the ammonia iron catalyst, which maintains its nanostructure (mean crystallite size and porosity) over a period of more than 15 years in an ammonia synthesis reactor (temperatures up to $500^{\circ} \mathrm{C}$ ).
The ability of the catalyst to maintain its nanostructure at a relatively high process temperature cannot be explained by the decay of diffusion effects. It has been observed $[8,9]$ that the surface structure of platinum gauze wires changes during catalytic ammonia oxidation. Consequently, surface development of the catalyst takes place as a result of interactions between the platinum and the gas phase.

Classical methods for describing the complex interactions between gas and solid phases are based on certain simplifications. In Langmuir's description of adsorption, only the equilibrium between the gas phase and the solid surface was considered [10]. He omitted the equilibrium between the substance adsorbed on the surface and the substance dissolved in the bulk. In the case of segregation, McLean [11] considered the equilibrium between the substances dissolved in the bulk and that segregated on the solid surface. However, he did not take into consideration the equilibrium between substances segregated on the solid surface and substances desorbed into the gas phase. In both studies, the possibilities of surface area modifications and the change in the coverage degree were not taken into account. 


\section{Results and Discussion}

To determine if materials with an extended surface exist in a state of chemical equilibrium, let us consider a two component system in which the surface of each individual crystallite (substance A) is covered with substance B (chemisorption) to a given degree, $\theta>0$. The coverage degree is defined as a ratio of number of occupied surface atoms to total number of adsorption sites. Let us assume that compound A-B is not formed, but a solution of B dissolved in A is formed. The equilibrium is not only limited to a balance between the surface and the volume of the crystallite, but it also includes the equilibrium of the covered and uncovered surface atoms and the equilibrium between B present on the crystallite's surface and in the gas phase:

$$
\mathrm{A}(\mathrm{B})_{\text {bulk }} \stackrel{K_{\text {bulk }}}{\longleftrightarrow} \mathrm{A}_{\text {surf }} \stackrel{K_{\text {surf }}}{\longleftrightarrow} \mathrm{A}(\mathrm{B})_{\text {surf }} \stackrel{K_{\text {gas }}}{\longleftrightarrow} \mathrm{B}_{\text {gas }} \text {. }
$$

The relationship between occupied free adsorption sites is determined by the coverage degree. In equilibrium, the coverage degree is unambiguously determined and results from the conditions that occur in that state. Thus, the equilibrium between covered and uncovered atoms means that there is a particular coverage degree observed in the system in question when the equilibrium state holds.

In the equilibrium expression above, the chemical potentials, $\mu$, of substances contained in the system are equal to one another. These potentials are related to the equilibrium constants, $K$, as follows:

$$
\begin{gathered}
K_{\text {surf }}=\frac{X_{\mathrm{A}(\mathrm{B}) \text {,surf }}}{X_{\mathrm{A}, \text { surf }}}, \\
K_{\text {bulk }}=\frac{X_{\mathrm{A}, \text { bulk }}}{X_{\mathrm{A}, \text { surf }}}, \\
K_{\text {gas }}=\frac{X_{\mathrm{B}, \text { gas }}}{X_{\mathrm{A}(\mathrm{B}) \text {,surf }}} .
\end{gathered}
$$

Molar concentration, $X$, can be calculated assuming that the total number of moles of atoms, $n$, is a sum of the moles of atoms of substance $\mathrm{A}, n_{\mathrm{A}}$, and substance $\mathrm{B}, n_{\mathrm{B}}$. For a single crystallite, the surface consisting of $n_{\mathrm{A} \text {,surf }}$ moles of atoms with unsaturated chemical bonds and $n_{\mathrm{A}(\mathrm{B}) \text {, surf }}$ moles of atoms with saturated bonds was considered separately. The crystallite bulk contains $n_{\mathrm{A} \text {,bulk }}$ moles of atoms. The number of moles of $\mathrm{B}$ atoms is the sum of the moles of $\mathrm{B}$ dissolved in the crystallite $\left(n_{\mathrm{B} \text {,bulk }}\right)$, the moles of $\mathrm{B}$ covering its surface, $n_{\mathrm{B} \text {,surf }}$, and the moles of $\mathrm{B}$ in the gas phase, $n_{\mathrm{B}, \mathrm{gas}}$. The degree of surface coverage of the crystallites, $\theta$, is defined as the ratio of the number of moles of $n_{\mathrm{A}(\mathrm{B}) \text {,surf }}$ to the total number of moles of $n_{\mathrm{A} \text {,surf }}$ and $n_{\mathrm{A}(\mathrm{B}) \text {,surf }}$. The molar concentration of substance $\mathrm{B}$ on the surface of the crystallite, $X_{\mathrm{A}(\mathrm{B}) \text {,surf }}$, can be changed by the simultaneous processes of adsorption and segregation (Figure 1).

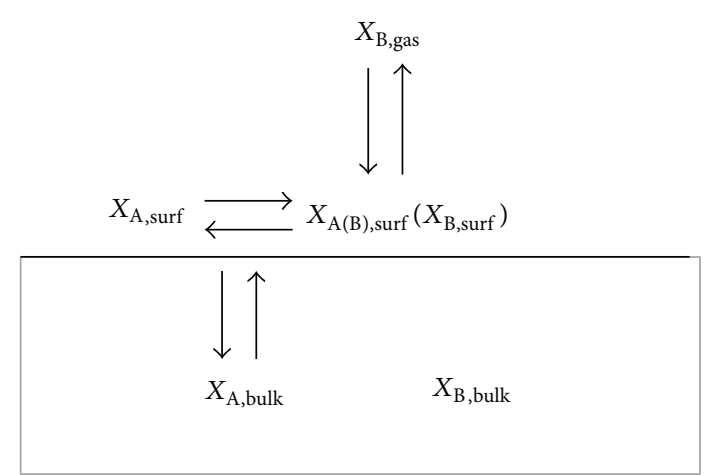

Figure 1: Scheme of the model system.

The specific surface area can be expressed as the ratio of the molar concentration of surface atoms to the total number of atoms in the crystallite:

$$
S=\zeta \frac{X_{\mathrm{A}, \text { surf }}+X_{\mathrm{A}(\mathrm{B}) \text {,surf }}}{X_{\mathrm{A}, \text { bulk }}+X_{\mathrm{A}, \text { surf }}+X_{\mathrm{A}(\mathrm{B}) \text {,surf }}}\left[\mathrm{m}^{2} \cdot \mathrm{g}^{-1}\right] \text {, }
$$

where the constant, $\zeta=\{(S / V) \cdot 1 / \rho\}$ ( $\rho$ is the density in $\left.\mathrm{g} \cdot \mathrm{m}^{-3}\right)$, is dependent on the substance.

Similar to Langmuir, it was assumed that there are no interactions between $\mathrm{B}$ atoms in the system. The surface energy corresponding to the clean surface of crystallite $A$ $(\theta=0)$ is $\gamma_{\mathrm{A}}$, and the surface energy for a surface completely covered with $\mathrm{B}$ atoms $(\theta=1)$ is equal to $\gamma_{\mathrm{A}(\mathrm{B})}$. Atoms with saturated and unsaturated bonds that are located on the surface of the crystallite are not energetically equivalent $\left(\gamma_{\mathrm{A}} \neq\right.$ $\left.\gamma_{\mathrm{A}(\mathrm{B})}\right)$. The difference between them is equal to the change in free energy, $\Delta G_{\text {surf }}$. Moreover, different crystallographic planes (edges, facets etc.), existing within clean and covered part of surface, have different surface energy values marked with subscript " $i$ " in equations below. Therefore, the surface energies associated with a clean surface and a completely covered surface can be expressed as follows:

$$
\begin{gathered}
\gamma_{\mathrm{A}}=\frac{\sum S_{\mathrm{A}, i} \gamma_{\mathrm{A}, i}}{S_{\mathrm{A}}}, \\
\gamma_{\mathrm{A}(\mathrm{B})}=\frac{\sum S_{\mathrm{A}(\mathrm{B}), i} \gamma_{\mathrm{A}(\mathrm{B}), i}}{S_{\mathrm{A}(\mathrm{B})}} .
\end{gathered}
$$

The faceting process is substantially associated with the process of edges formation. However, in the model calculations the values of surface energy for covered and uncovered parts of surface were taken as average for the whole part of surface (covered or uncovered) without specifying energy ratios associated with particular surface structures (edges, facets, etc.).

On the surface of a pure crystallite, there are only uncovered atoms $\left(S=S_{\mathrm{A}}\right)$, resulting in a crystallite with a high surface energy. Partial coverage $(0<\theta<1)$ of the crystallite's surface leads to a reduction of its surface energy as a result of saturation of a portion of the free bonds on the surface of the crystallite $\left(\gamma_{\mathrm{A}}>\gamma_{\mathrm{A}(\mathrm{B})}\right)$. Assuming that $\theta_{1}$ is less 
than $\theta_{2}$, the reduction of the surface energy can be expressed as follows:

$$
\gamma_{\mathrm{A}}>\gamma_{\mathrm{A}}\left(1-\theta_{1}\right)+\gamma_{\mathrm{A}(\mathrm{B})} \theta_{1}>\gamma_{\mathrm{A}}\left(1-\theta_{2}\right)+\gamma_{\mathrm{A}(\mathrm{B})} \theta_{2} .
$$

The total value of the surface energy, $\gamma$, which corresponds to the energy minimum (at equilibrium state), results from the tendency of the system to reduce its specific surface area and to increase the degree of surface coverage:

$$
\gamma=\gamma_{\mathrm{A}}(1-\theta)+\gamma_{\mathrm{A}(\mathrm{B})} \theta .
$$

At the moment of preparation of nanocrystalline substance, the whole surface of every nanocrystallite is fully covered by promoters' atoms, $\theta=1$, and crystallites are small (diameter of tens of nanometer). This is the global minimum of the energy of system in question. To change (decrease) the value of the coverage degree one has to add some energy to the system. This means that we move up from the point of minimum energy because the system gets some additional energy. However, at that new situation, a new state of equilibrium holds, which corresponds to new process conditions and one deals with new local minimum energy.

From a thermodynamic standpoint, each process reaches equilibrium in an infinitely long time. As a result of a rise in temperature, an increase in the diffusion rate is observed, which can alter the equilibration rate of the system. These changes allow the system to reach a state of thermodynamic equilibrium in a realistic experimental time. Therefore, obtaining a stable nanostructure results from achieving an energy minimum, that is, a state of equilibrium, rather than the inhibition of diffusion.

Chemisorption of substances on the surface of the crystallite is associated with a specific energetic effect that can cause an increase in the energy of the system resulting in a temperature increase. According to the Le ChatelierBraun principle, the higher the temperature of a system is, the less likely an additional temperature increases. This is due to consecutive portions of supplied energy. Therefore, the process can be expected to occur under isothermaladiabatic conditions. The energy released during the surface enrichment of a solid with substance $B$ can be used to change the crystallites' surface area. The surface coverage degree, $\theta$, is dependent on enrichment processes (e.g., adsorption or segregation) and can be calculated using the Langmuir [10] and McLean [11] equations:

$$
\begin{gathered}
\frac{\theta}{1-\theta}=p \exp \left(-\frac{\Delta G_{\mathrm{ads}}}{R T}\right), \\
\frac{\theta}{1-\theta}=\frac{X_{\mathrm{B}, \text { bulk }}}{1-X_{\mathrm{B}, \mathrm{bulk}}} \exp \left(-\frac{\Delta G_{\mathrm{seg}}}{R T}\right) .
\end{gathered}
$$

The energy balance of the system is expressed by the following equation:

$$
\begin{aligned}
S_{\mathrm{A}(\mathrm{B})} \mathrm{Q} \theta+S_{\mathrm{A}} \gamma_{\mathrm{A}}= & S_{\mathrm{A}(\mathrm{B})}\left(\gamma_{\mathrm{A}}(1-\theta)+\gamma_{\mathrm{A}(\mathrm{B})} \theta\right) \\
& +\left(S_{\mathrm{A}(\mathrm{B})}-S_{\mathrm{A}}\right) \gamma_{\mathrm{A}} .
\end{aligned}
$$

The left side of (8) defines the surface energy for a solid that has a surface consisting only of uncovered atoms and an energy, $Q$, that is released during the interactions between substances $A$ and $B$. The right side of (8) is the sum of the surface energy of phase A, which has a surface partly covered with particles of substance $B$, and the energy needed to create a new, additional surface. Based on this balance, the degree of specific surface development of a nanocrystalline substance is expressed as the $S_{\mathrm{A}(\mathrm{B})} / S_{\mathrm{A}}$ ratio, which can be expressed in a general equation of the following form:

$$
\begin{aligned}
\frac{S_{\mathrm{A}(\mathrm{B})}}{S_{\mathrm{A}}} & =\frac{2 \gamma_{\mathrm{A}}}{2 \gamma_{\mathrm{A}}+\theta\left(\Delta G_{\text {surf }}+\Delta G_{\text {seg }}\right)} \\
& =\frac{2 \gamma_{\mathrm{A}}}{2 \gamma_{\mathrm{A}}-\theta\left[R T \ln \left(K_{\text {surf }} K_{\text {bulk }}\right)\right]} .
\end{aligned}
$$

The specific surface area of nanocrystalline substances may vary as a result of changes in the size and number of crystallites and in the surface structure (faceting). The balance between the above-mentioned factors is determined by the tendency of the system to achieve an energy minimum. In the process of faceting, new surfaces are formed on the crystallites by crystallographic planes with different orientations, although the size and number of crystallites remain unchanged. The surface energies $\gamma_{\mathrm{A}}$ and $\gamma_{\mathrm{A}(\mathrm{B})}$ change according to (4a) and (4b). For the sake of clarity, a system containing substances $\mathrm{A}$ and $\mathrm{B}$ was studied under isothermal conditions, and the concentration of $X_{\mathrm{B} \text {,bulk }}$ was varied while taking into account the simultaneously occurring processes of adsorption and segregation. Regardless of the conditions, the same crystallographic planes are exhibited on the surface of crystallites, and the surface energies $\left(\gamma_{\mathrm{A}}, \gamma_{\mathrm{A}(\mathrm{B})}\right)$ remain constant during the process. At a given temperature, development of the surface (from $S_{\mathrm{A}}$ to $S_{\mathrm{A}(\mathrm{B})}$ ) may occur as a result of an increase in the number of crystallites due to a reduction in their size $\left(d_{\mathrm{A}(\mathrm{B})}<d_{\mathrm{A}}\right)$ during the process of recrystallisation. All the calculations were carried out on the basis of (7a), (7b), and (9), and the results obtained are shown in Figure 2. Modeling shown in the present paper is based on the laboratory experience concerning iron ammonia synthesis catalyst [12-16].

For various concentrations of $X_{\mathrm{B}, \text { bulk }}$, the equilibrium values of the surface coverage degree and gas pressure were determined. The consequence of increasing the surface coverage degree is an increase in surface degree development.

Calculations for an isolated system of A and B in which the equilibrium was achieved at various temperatures were also carried out. At a given temperature, $T_{1}$, the effect of adsorption (assuming no desorption) and segregation on coverage degree, $\theta>0$, was determined for the surface of crystallite A with a diameter, $d_{\mathrm{A}}$ (for simplicity it was assumed that all crystallites had the same size), a volume, $V_{\mathrm{A}}$, and a minimum surface area, $S_{\mathrm{A}}$. A change in the surface area of crystallites calculated using (9) results from an increase in the surface coverage of the crystallite with substance $B$. Substituting the value for the surface area of the crystallite, $S_{\mathrm{A}(\mathrm{B})}$, determined from (9) into (7a) or (7b) allows for a new surface coverage degree to be calculated. Then, from (9), a change in the surface area of crystallites was recalculated. Iterations were performed until the surfaces calculated from 


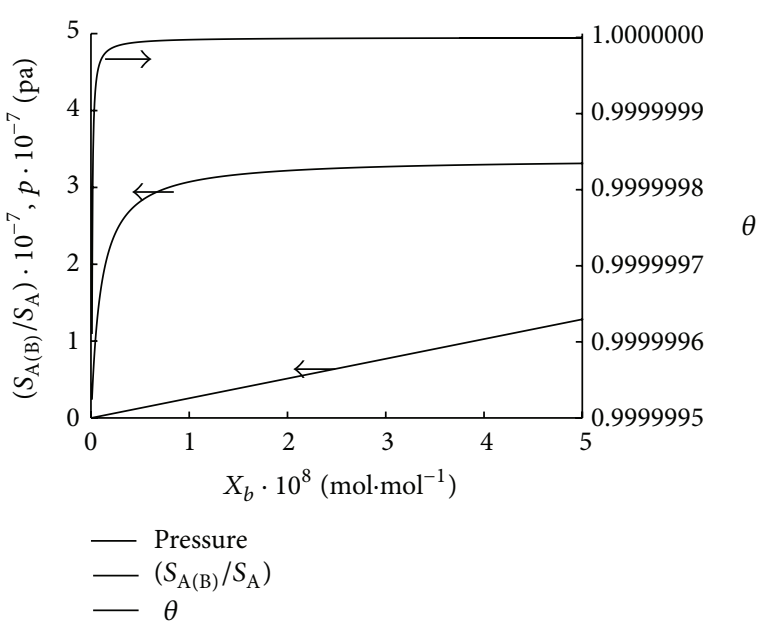

Figure 2: Dependence of surface development degree $\left(S_{\mathrm{A}(\mathrm{B})} / S_{\mathrm{A}}\right)$, surface coverage degree $(\theta)$, and pressure $(p)$ of substance $B$ in the gas phase on bulk concentration of substance $\mathrm{B}\left(X_{\mathrm{B}}\right)$. Calculations performed for data as follows: $\Delta G_{\text {seg }}=-180 \mathrm{~kJ} \cdot \mathrm{mol}^{-1}, T=573 \mathrm{~K}$, $K=100, \gamma_{\mathrm{A}}=170 \mathrm{~kJ} \cdot \mathrm{mol}^{-1}, \gamma_{\mathrm{A}(\mathrm{B})}=10 \mathrm{~kJ} \cdot \mathrm{mol}^{-1}$.

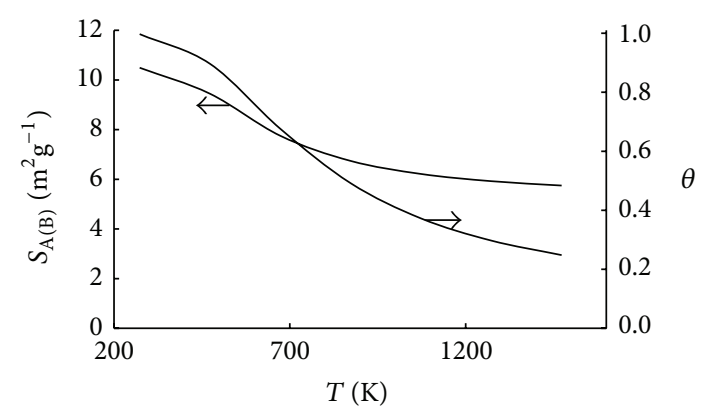

FIgURE 3: Dependence of the specific surface area $S_{\mathrm{A}(\mathrm{B})}$ on temperature, where $\Delta G_{\text {seg }}=-20 \mathrm{~kJ} \cdot \mathrm{mol}^{-1}, \gamma_{\mathrm{A}}=200 \mathrm{~kJ} \cdot \mathrm{mol}^{-1}, \gamma_{\mathrm{A}(\mathrm{B})}=$ $10 \mathrm{~kJ} \cdot \mathrm{mol}^{-1}$ and $X_{\mathrm{B}}=n_{\mathrm{B}} / n=0.0625 \mathrm{~mol} \cdot \mathrm{mol}^{-1}$.

(7a), (7b), and (9) were equal. The same calculation procedure was used to determine the specific surface area of crystallites at temperatures $T>T_{1}$. The results of these calculations are shown in Figure 3.

Single component materials tend to achieve an energy minimum by reducing the specific surface area and thus reducing the energy of the system. From a thermodynamic standpoint, only monocrystals can be in equilibrium. The internal energy of complex systems with at least two substances depends on the surface energy of covered and uncovered atoms and the energy of bonds between chemisorbed atoms and surface atoms of the crystallites. The system reaches its minimum energy as a result of compensating the surface energy through the creation of bonds between surface atoms of the two substances. If processes are not limited by diffusion effects and are connected with a change in the concentration of chemisorbed atoms under isothermal-adiabatic conditions, the energy released during these processes can be used to change the surface area of crystallites.
Under real conditions, the size of the surface area is determined not only by thermodynamic parameters but also by the kinetic aspects. Nanocrystalline materials with highly developed, stable surfaces can be obtained only if the growth of crystallites is the limiting step during the preparation and the proper conditions are provided for covering the surface of nanocrystallite A with substance B. As a result of the process being conducted this way, a nanostructured material with a minimum energy at a given size of crystallites is obtained.

From a thermodynamic point of view, the reduction of the surface area is associated with a decrease in the surface coverage degree and thus with a necessity to provide energy to the system to remove chemisorbed atoms. An increase in the temperature of a nanocrystalline substance above the preparation temperature results in the formation of a new state of equilibrium defined by larger sizes and a lower number of crystallites, resulting in a smaller specific surface area. In practice, the reverse process is not observed despite the use of a lower temperature. The reduction of the size of crystallites cannot be achieved due to the slow rate of this process. Lowering the temperature does not change the size and number of crystallites, even though an increase in the degree of fineness is thermodynamically possible. A system reaches a state of equilibrium at a temperature equal to or greater than the annealing temperature. At temperatures below the maximum annealing temperature, one can only discuss the balance between the gas phase and the surface, whose structure may change through the faceting processes due to diffusion of surface atoms. The occurrence of the phenomenon in glasses, gels, supersaturated solid solutions, and implanted materials has been previously described [4].

\section{Conclusions}

The results presented shed new light on the structure of nanomaterials. According to common opinion, materials consisting of nanometer-sized crystallites are far away from thermodynamic equilibrium. Under our defined conditions, these materials can be in thermodynamic equilibrium. The stable structure of these materials is the result of maintaining a state of thermodynamic equilibrium.

\section{Conflict of Interests}

The authors declare that there is no conflict of interests regarding the publication of this paper.

\section{Acknowledgment}

The scientific work was financed from the budgetary resources for the project "Cobalt catalysts for energy-efficient process of ammonia synthesis," Project no. PBS2/Al/13/2014.

\section{References}

[1] F. Wakai and F. Aldinger, "Sintering forces in equilibrium and non-equilibrium states during sintering of two particles," Science and Technology of Advanced Materials, vol. 5, no. 4, pp. 521-525, 2004. 
[2] F. Wakai, M. Yoshida, Y. Shinoda, and T. Akatsu, "Coarsening and grain growth in sintering of two particles of different sizes," Acta Materialia, vol. 53, no. 5, pp. 1361-1371, 2005.

[3] S. S. Dukhin, J. Yang, R. N. Dave, and R. Pfeffer, "Deactivated sintering by particle coating: the significance of static and dynamic surface phenomena," Colloids and Surfaces A: Physicochemical and Engineering Aspects, vol. 235, no. 1-3, pp. 83-99, 2004.

[4] H. Gleiter, "Nanostructured materials: basic concepts and microstructure," Acta Materialia, vol. 48, no. 1, pp. 1-29, 2000.

[5] L. Dazhuang and H. Wensheng, "Determination of equilibrium dispersion of Pt on $\mathrm{Al}_{2} \mathrm{O}_{3}$ support in sintering process," Reaction Kinetics and Catalysis Letters, vol. 71, no. 2, pp. 295-300, 2000.

[6] C. H. Bartholomew, "Mechanisms of catalyst deactivation," Applied Catalysis A: General, vol. 212, no. 1-2, pp. 17-60, 2001.

[7] A. Monzón, T. F. Garetto, and A. Borgna, "Sintering and redispersion of $\mathrm{Pt} / \gamma-\mathrm{Al}_{2} \mathrm{O}_{3}$ catalysts: a kinetic model," Applied Catalysis A: General, vol. 248, no. 1-2, pp. 279-289, 2003.

[8] Z. Rdzawski, L. Ciura, and B. Nikiel, "Metallographic examination of catalyst gauzes and catchment gauzes from platinum and palladium alloys," Journal of Materials Processing Technology, vol. 53, no. 1-2, pp. 319-329, 1995.

[9] J. Schroeder, J. Jarmakowicz, and J. Zabrzeski, “The modifying effect of added metals on the surface of platinum catalyst used for ammonia oxidation," Journal of Catalysis, vol. 82, no. 1, pp. 66-76, 1983.

[10] I. Langmuir, "The adsorption of gases on plane surfaces of glass, mica and platinum," The Journal of the American Chemical Society, vol. 40, no. 9, pp. 1361-1403, 1918.

[11] J. Benard, Ed., Adsorption on Metal Surfaces. Studies in Surface Science and Catalysis, Elsevier, Amsterdam, The Netherlands, 1983.

[12] W. Arabczyk, I. Jasińska, and K. Lubkowski, "The surface properties of iron catalyst for ammonia synthesis," Reaction Kinetics and Catalysis Letters, vol. 83, no. 2, pp. 385-392, 2004.

[13] I. Jasińska and W. Arabczyk, "Kinetic studies of the recrystallization process of iron catalyst for ammonia synthesis," Chemical Papers, vol. 59, no. 6, pp. 496-499, 2005.

[14] R. Pelka and W. Arabczyk, "A new method for determining the nanocrystallite size distribution in systems where chemical reaction between solid and a gas phase occurs," Journal of Nanomaterials, vol. 2013, Article ID 645050, 6 pages, 2013.

[15] D. Moszyński, I. Moszyńska, and W. Arabczyk, "The transformation of $\alpha$-Fe into $\gamma^{\prime}-\mathrm{Fe}_{4} \mathrm{~N}$ in nanocrystalline $\mathrm{Fe}-\mathrm{N}$ system: influence of Gibbs-Thomson effect," Applied Physics Letters, vol. 103, no. 25, Article ID 253108, 2013.

[16] D. Moszyński, "Nitriding of nanocrystalline iron in the atmospheres with variable nitriding potential," The Journal of Physical Chemistry C, vol. 118, pp. 15440-15447, 2014. 

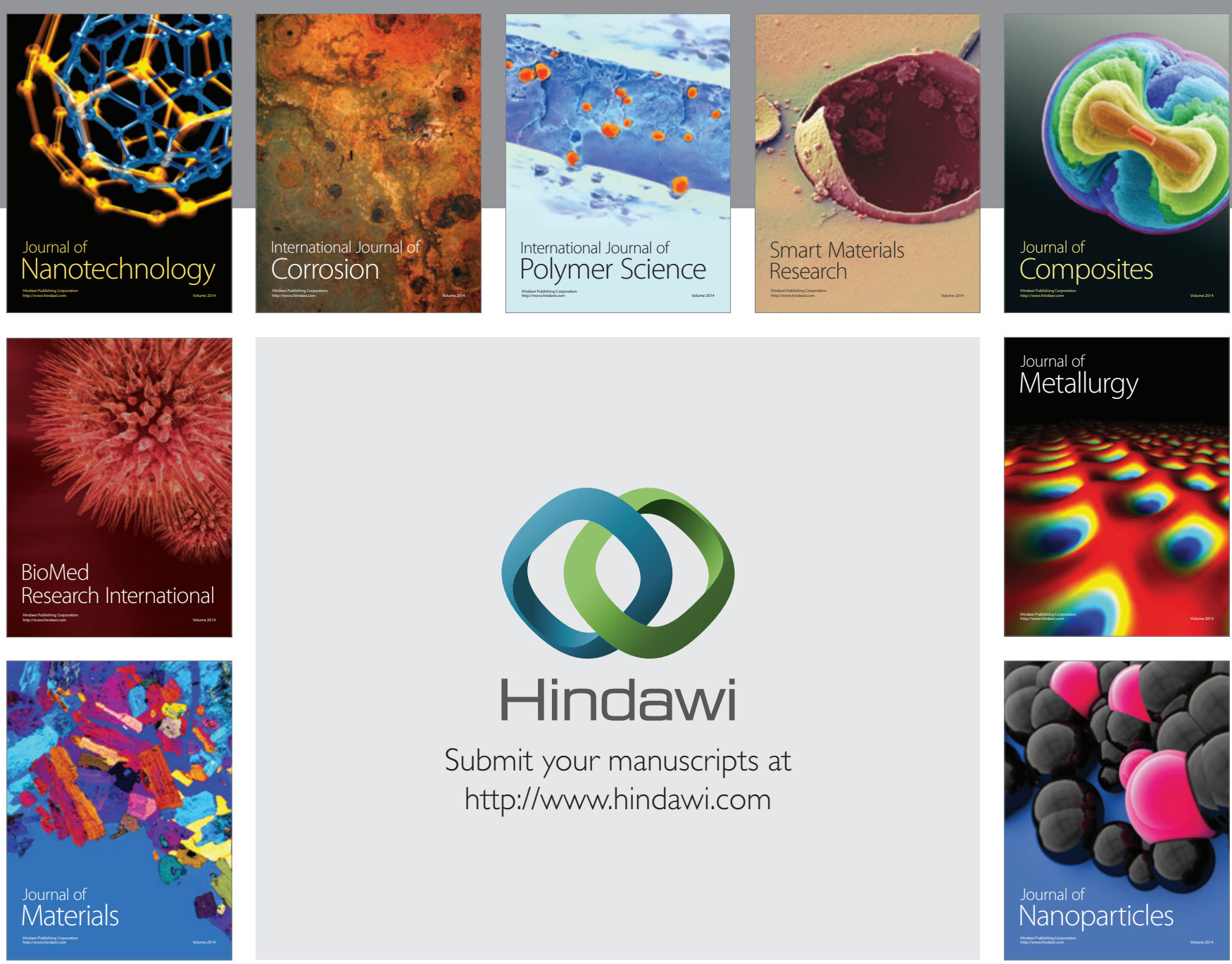

Submit your manuscripts at http://www.hindawi.com
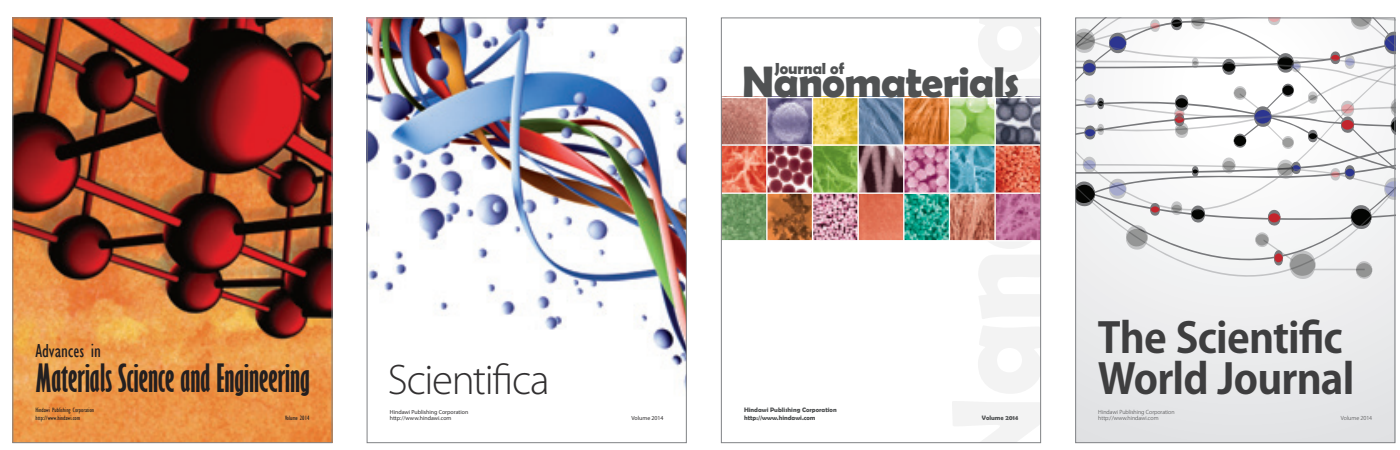

\section{The Scientific World Journal}
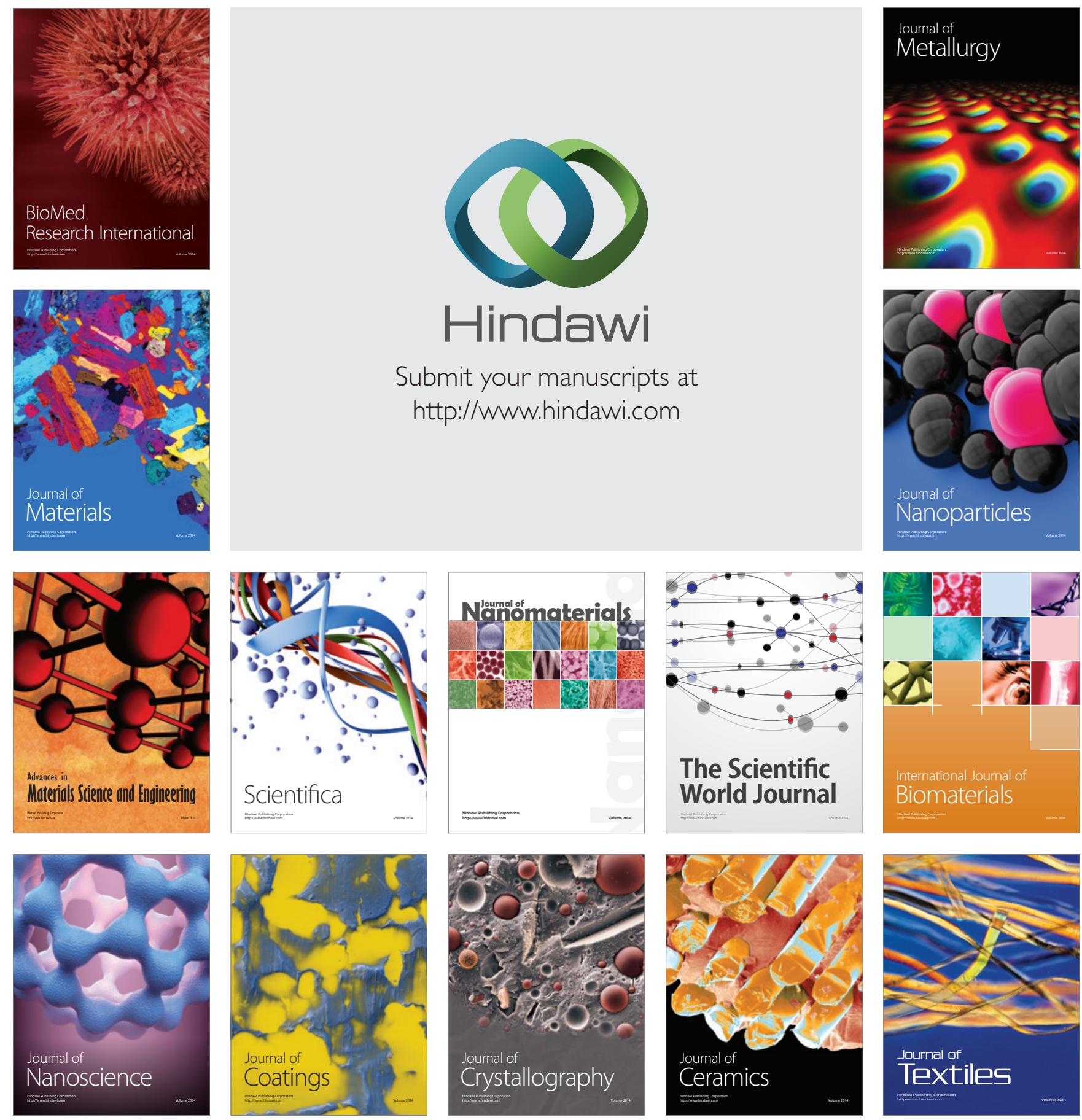